\title{
Sleep Differentially Affects Early and Late Neuronal Responses to Sounds in Auditory and Perirhinal Cortices
}

\author{
다 Yaniv Sela, ${ }^{1}$ Aaron Joseph Krom, ${ }^{2,3}$ Lottem Bergman, ${ }^{3}$ Noa Regev, ${ }^{3}$ and $\odot$ Yuval Nir ${ }^{1,3}$ \\ ${ }^{1}$ Sagol School of Neuroscience, Tel Aviv University, Tel Aviv, Israel, 69978, ${ }^{2}$ Department of Anesthesiology and Critical Care Medicine, Hadassah-Hebrew \\ University Medical Center, Hebrew University-Hadassah School of Medicine, Jerusalem, Israel, 91120, and ${ }^{3}$ Department of Physiology and Pharmacology, \\ Sackler School of Medicine, Tel Aviv University, Tel Aviv, Israel, 69978
}

A fundamental feature of sleep is reduced behavioral responsiveness to external events, but the extent of processing along sensory pathways remains poorly understood. While responses are comparable across wakefulness and sleep in auditory cortex (AC), neuronal activity in downstream regions remains unknown. Here we recorded spiking activity in 435 neuronal clusters evoked by acoustic stimuli in the perirhinal cortex (PRC) and in AC of freely behaving male rats across wakefulness and sleep. Neuronal responses in AC showed modest $(\sim 10 \%)$ differences in response gain across vigilance states, replicating previous studies. By contrast, PRC neuronal responses were robustly attenuated by $47 \%$ and $36 \%$ during NREM sleep and REM sleep, respectively. Beyond the separation according to cortical region, response latency in each neuronal cluster was correlated with the degree of NREM sleep attenuation, such that late $(>40 \mathrm{~ms})$ responses in all monitored regions diminished during NREM sleep. The robust attenuation of late responses prevalent in PRC represents a novel neural correlate of sensory disconnection during sleep, opening new avenues for investigating the mediating mechanisms.

Key words: auditory; NREM; perirhinal; REM; single-unit; sleep

Significance Statement

Reduced behavioral responsiveness to sensory stimulation is at the core of sleep's definition, but it is still unclear how the sleeping brain responds differently to sensory stimuli. In the current study, we recorded neuronal spiking responses to sounds along the cortical processing hierarchy of rats during wakefulness and natural sleep. Responses in auditory cortex only showed modest changes during sleep, whereas sleep robustly attenuated the responses of neurons in high-level perirhinal cortex. We also found that, during NREM sleep, the response latency predicts the degree of sleep attenuation in individual neurons above and beyond their anatomical location. These results provide anatomical and temporal signatures of sensory disconnection during sleep and pave the way to understanding the underlying mechanisms.

\section{Introduction}

Reduced behavioral responses to external sensory stimuli are an essential definition of sleep (Carskadon and Dement, 2011) and are ubiquitous across the animal kingdom (Cirelli and Tononi, 2008). Yet, how sleep affects the responses along sensory pathways remains poorly understood. During sleep, external stimuli rarely affect perception (Nir and Tononi, 2010), but it is also clear that

Received May 22, 2019; revised Dec. 31, 2019; accepted Jan. 7, 2020.

Author contributions: Y.S., A.J.K., and Y.N. designed research;Y.S., A.J.K., L.B., and N.R. performed research; Y.S. analyzed data; Y.S. wrote the first draft of the paper; Y.S., A.J.K., L.B., N.R., and Y.N. edited the paper; Y.S. and Y.N. wrote the paper.

This work was supported by Israel Science Foundation Grant 1326/15 and 51/11 (I-CORE Cognitive Sciences) and the Adelis Foundation to Y.N., Israel Science Foundation Grant 762/16 and European Society of Anesthesia Young Investigator Start-up Grant to A.J.K., and Azrieli Foundation Azrieli Fellowship Award to Y.S. We thank Israel Nelken for suggesting focusing on perirhinal cortex as high-order region; Hanna Hayat and Amit Marmelshtein for input; and all members of Y.N. laboratory for discussions.

The authors declare no competing financial interests.

Correspondence should be addressed to Yuval Nir at yuvalnir.tau@gmail.com.

https://doi.org/10.1523/JNEUROSCI.1186-19.2020

Copyright $\odot 2020$ the authors some discriminative sensory processing persists (Andrillon et al., 2016). Surprisingly, only few studies investigated sensory responses during natural sleep at the neuronal level (Hennevin et al., 2007). Early studies (Gücer, 1979; Livingstone and Hubel, 1981) contributed to the "thalamic gating" model (Steriade and McCarley, 1990), proposing that thalamic burst-mode activity and sleep spindle oscillations disrupt signal propagation to the cerebral cortex during sleep (McCormick and Bal, 1994; Steriade, 2003). However, this view was challenged by sleep attenuation of responses in the olfactory cortex that are not relayed through thalamus (Murakami et al., 2005). In addition, several recent studies in the auditory system showed that responses in the primary and secondary auditory cortex (AC) are comparable across wakefulness and natural sleep (Peña et al., 1999; Edeline et al., 2001; Issa and Wang, 2008; Nir et al., 2015), even at times when sleep spindles occur (Sela et al., 2016).

Do sounds effectively modulate responses in downstream association cortex during sleep? On one hand, hippocampaldependent memory consolidation can be promoted via targeted memory reactivation, which re-presents sounds that were used as 
training cues or context during sleep (Oudiette and Paller, 2013). Targeted memory reactivation has been shown to influence hippocampal activity during sleep (Rasch et al., 2007; Rothschild et al., 2017), suggesting that sounds can modulate activity in association cortices. On the other hand, noninvasive imaging in humans suggests that, during sleep, auditory responses downstream from auditory cortex are attenuated: studies using MEG (Kakigi et al., 2003; Strauss et al., 2015), EEG (Makov et al., 2017), and fMRI (Portas et al., 2000) suggest comparable responses across vigilance states in early sensory regions and robust attenuation in association cortex. However, investigation of sensory responses downstream from sensory cortical regions at the level of neuronal spiking activity is still missing.

We hypothesized that the perirhinal cortex (PRC) constitutes a high-order cortical region that may show robust attenuation of sensory responses during sleep. The PRC is a multimodal region downstream to several primary sensory cortices, where information converges from different sensory modalities to support higher brain functions, including perception and recognition memory (Kealy and Commins, 2011). Moreover, it has been shown to contain intrinsic inhibitory mechanisms that affect information flow to the hippocampus (de Curtis and Paré, 2004; Pelletier et al., 2004). PRC is located further downstream from the suprarhinal auditory field (Profant et al., 2013; Lee et al., 2016). While perirhinal neurons reliably respond to sounds during wakefulness (Allen et al., 2007; Furtak et al., 2007), separate studies during anesthesia did not reveal significant auditory responses as one moves ventrally toward rhinal fissure (Doron et al., 2002). For the above reasons, we set out to directly compare auditory responses in PRC neurons across wakefulness and natural sleep. We also compared perirhinal response profiles with simultaneously recorded neurons in AC. We find that PRC neurons robustly attenuate their auditory responses during sleep, in contrast to AC neurons, which are minimally affected by vigilance state. In addition, we find that the response latency of each neuronal cluster (typically $<20 \mathrm{~ms}$ in $\mathrm{AC}$ and $>40 \mathrm{~ms}$ in PRC) is correlated with the degree of NREM sleep attenuation, and better predicts attenuation than does cortical region.

\section{Materials and Methods}

\section{Animals}

Experiments were performed in 8 Long-Evans and 2 Wistar adult male rats $(250-350 \mathrm{~g})$ individually housed in transparent Perspex cages with food and water available ad libitum. Ambient temperature was kept at $20^{\circ} \mathrm{C}-24^{\circ} \mathrm{C}$ and light-dark cycles of $12 / 12 \mathrm{~h}$ at 10:00 A.M./10:00 P.M. All experimental procedures, including animal handling, surgery, and experiments, followed the National Institutes of Health's Guide for the care and use of laboratory animals and were approved by the Institutional Animal Care and Use Committee of Tel Aviv University.

\section{Surgery and electrode implantation}

Animals were anesthetized using isoflurane (4-5\% induction, $1 \%-2 \%$ maintenance) and placed in a stereotactic frame (David Kopf Instruments) while maintaining body temperature $\sim 37^{\circ} \mathrm{C}$ using a closed-loop heating pad system (Harvard Apparatus). The head was shaved, and Viscotear gel was applied to protect the eyes. After exposing and cleaning the skull, frontal and parietal screws ( $1 \mathrm{~mm}$ in diameter) were placed over the left hemisphere for EEG recording. Two screws were placed above the cerebellum as reference and ground, and an additional anchor screw above the right frontal lobe for stabilization. Two single-stranded stainless-steel wires were inserted to either side of neck muscles to measure EMG (see Fig. 1A). EEG and EMG wires were soldered onto a headstage connector (Omnetics Connector). Dental cement was used to cover all screws and EEG/EMG wires.

A small craniotomy was performed over the right hemisphere, and the dura was reflected under microscopic control. Multichannel microwire arrays (each wire: $50 \mu \mathrm{m}$ diameter, $45^{\circ}$ tip angle) were implanted (Tucker-Davis Technologies). The arrays consisted of either 16 wires (2 rows $\times 8$ channels, $9.5 \mathrm{~mm}$ long) or 32 wires ( 4 rows $\times 8$ channels, two short $7.5 \mathrm{~mm}$ rows and two long $9.5 \mathrm{~mm}$ rows), with $375 \mu \mathrm{m}$ mediallateral separation between rows and $250 \mu \mathrm{m}$ anterior-posterior separation within each row. Implantation was made either diagonally (insertion point P: $3.6 \mathrm{~mm}$, L: $4.2 \mathrm{~mm}$ relative to bregma and inserted to a depth of $8 \mathrm{~mm})$ in angle of $22^{\circ}(n=3)$ or vertically (P: $3.6 \mathrm{~mm}, \mathrm{~L}: 6.65$ $\mathrm{mm}, \mathrm{D}: 7.4 \mathrm{~mm}$ relative to bregma), after the temporalis muscle was gently separated and retracted from the bone $(n=7$; see Fig. $1 B)$. After implantation, the craniotomy was covered with silicon gel (Kwik-Sil; World Precision Instruments) and the electrode array was fixed in place with Fusio (Pentron).

\section{Perioperative analgesia and antibiotic prophylaxis}

After anesthesia induction, carprofen $(5 \mathrm{mg} / \mathrm{kg})$ and dexamethasone $(0.5$ $\mathrm{mg} / \mathrm{kg}$ ) were administrated intraperitoneally, and the surgical site was infiltrated subcutaneously with lignocaine $(7 \mathrm{mg} / \mathrm{kg})$ before incision. Cefazolin $(20 \mathrm{mg} / \mathrm{kg}$ i.m.) was administered for surgical wound infection prophylaxis before incision, and chloramphenicol 3\% ointment applied at the end of surgery. Postoperative analgesia was provided by injecting buprenorphine systemically $(0.025 \mathrm{mg} / \mathrm{kg}$ s.c. $)$ as the rat started showing signs of awakening from anesthesia. In the days following surgery, dexamethasone $(1.3 \mathrm{mg} / \mathrm{kg})$ was given with food to reduce pain and inflammation around implanted electrodes.

\section{Histology}

At the end of each experiment, rats were deeply anesthetized (5\% isoflurane) and transcardially perfused with saline followed by $4 \%$ PFA. Brains were refrigerated in PFA for at least a week and sectioned in 50-60 $\mu \mathrm{m}$ serial coronal sections using a Vibrating Microtome (Leica Biosystems). Slices were stained with fluorescent cresyl violet (Rhenium).

\section{Electrophysiology and spike sorting}

All electrophysiological data were acquired using a RZ2 high performance processor (Tucker-Davis Technologies). Extracellular activity was continuously recorded from microwires and digitally sampled at 24.4 $\mathrm{kHz}$ (PZ2 amplifier, Tucker-Davis Technologies). We then applied a high-pass filter $(300-5000 \mathrm{~Hz}$, fourth-order zero-phase elliptic filter) for detecting action potentials (APs). Epidural EEG and EMG were preamplified (RA16LI, Tucker-Davis Technologies) and digitally sampled at $256.9 \mathrm{~Hz}$ (PZ2 amplifier, Tucker-Davis Technologies). Offline, EEG and EMG were resampled to $1000 \mathrm{~Hz}$ (MATLAB, The MathWorks) and filtered $(0.5-300 \mathrm{~Hz}$ for EEG, $10-100 \mathrm{~Hz}$ for EMG, fourth-order zerophase Butterworth filters) for offline sleep scoring and power spectrum analysis (see Fig. 2D). All data were synchronized with a video system (RV2, Tucker-Davis Technologies). Spike sorting was performed offline with "wave_clus" (Quiroga et al., 2004) using a detection thresholds around 5 SD (median $\{\mid$ data $\mid / 0.6745\}$ ) above the median activity (see Fig. $3 A$ ), and applying automatic superparamagnetic clustering of wavelet coefficients followed by manual refinement based on consistency of spike waveforms and interspike interval distribution (see Fig. $3 B, C$ ).

\section{Auditory stimulation}

All experiments were conducted in a sound-attenuation $(-55 \mathrm{~dB})$ chamber (H.N.A.). Sounds were synthesized in MATLAB, transduced to voltage signal (195 kHz sampling rate, RZ6, Tucker-Davis Technologies), passed through a zero-amplification speaker amplifier (SA1, TuckerDavis Technologies), and played free field via a magnetic ultrasonic speaker (MF1, Tucker-Davis Technologies) mounted $55 \mathrm{~cm}$ above the center of the cage (see Fig. 1E). Given the free-field setup, stimulus intensity at the animal's ears in each trial inevitably varies depending on precise position relative to speaker. We measured sound intensity (values below) with an ultrasonic calibration microphone (PCB Piezotronics, model 378C01) placed directly below the speaker in the center of the cage. During experiments, a wide battery of 36 sounds (500 ms each, except for a $1 \mathrm{~ms}$ click stimulus) were delivered, including the following: pure tone pips $(3,6,12,24$, and $48 \mathrm{kHz})$ at three different intensities (76, 82, $93 \mathrm{~dB}$ SPL); single octave band-passed white noise (centered on the same frequencies as tone pips); $40 \mathrm{~Hz}$ click trains (with 3 intensities as 
above); and single clicks. In addition, we presented behaviorally relevant sounds of prerecorded (Avisoft Bioacoustics; www.avisoft.com) ultrasonic rodent vocalizations (ultrasonic vocalization, 24,42 , and $66 \mathrm{kHz}$, and six types of $60 \mathrm{kHz}$ ), concatenated to produce 500-ms-long stimuli. Finally, in an attempt to generate behaviorally meaningless stimuli with near-identical physical features, three additional vocoded vocalizations were generated by taking the first 3 ultrasonic vocalizations above, identifying their principle frequency, and modulating a pure tone of this frequency using their original sound intensity envelopes.

\section{Experimental design}

Three days before the surgery, rats were moved with their home cage inside an acoustic chamber for habituation to the experimental environment. After surgery, a recovery of 1 week was allowed undisturbed for renormalization of the sleep/wake cycle. Next, 2-3 additional days were used for habituation to recording cable and to auditory stimulation. Experimental sessions lasted 5-9 h and started between 10:00-12:00 to maximize REM sleep trials in the late "lights-on" afternoon sleep hours. All 36 stimuli were presented in a pseudorandom order with an average interstimulus interval of $2.5 \pm 0.4 \mathrm{~s}$ (with jitter). There was a pause of 10 min after every cycle of 24 repetitions of all stimuli.

\section{Sleep scoring}

Sleep scoring was based on a combined manual examination of frontal and parietal EEG, EMG, and video data, using custom MATLAB software, which enabled precise marking of state transitions. Data were divided to six vigilance states (see Fig. 2A) as in Datta and Hobson (2000): (1) quiet wake: low-voltage high-frequency EEG, and high tonic EMG; (2) active wake: low-voltage high-frequency EEG, accompanied by phasic EMG activity and behavioral activity (e.g., eating, grooming or exploring) confirmed with video; (3) NREM sleep: high-amplitude slowwave activity and low tonic EMG activity; (4) light NREM sleep: transition state between wakefulness and NREM sleep, characterized by EEG slowing without obvious slow waves co-occurring with reduced EMG; (5) REM sleep: high-frequency wake-like frontal EEG co-occurring with theta activity in parietal EEG and flat EMG; and (6) NREM-REM transition: transition into REM sleep showing mixed EEG signatures of both NREM and REM sleep (e.g., weak theta in parietal EEG with spindle activity in frontal EEG). Subsequent analysis of auditory responses focused on quiet wakefulness, NREM sleep, and REM sleep and did not include periods of light NREM sleep ( $14 \pm 2.6 \%)$, NREM-REM transition periods ( $3 \pm 1.3 \%$ of data), active wake ( $13 \pm 4.8 \%$ of data), or intervals not matching these six states $(5 \pm 2.8 \%$ of data). Joint distributions of EMG levels and EEG high-/low-frequency power ratio (see Fig. $2 E$ ) were calculated based on $4 \mathrm{~s}$ epochs. Power ratio was defined as power $>25 \mathrm{~Hz}$ divided by power $<5 \mathrm{~Hz}$ in the frontal EEG.

\section{Analysis of auditory responses}

Spontaneous firing rate. Spontaneous activity of each neuronal cluster was defined as the averaged firing rate in the $500 \mathrm{~ms}$ before each auditory trial, calculated separately for each state.

Detection of significant responses. We detected time intervals of significant increased neuronal activity in responses to auditory stimuli as follows: First, each of $N$ trials was smoothed by convolution with a Gaussian kernel $(\sigma=8 \mathrm{~ms})$. Then, a one-tailed Mann-Whitney test compared, for each millisecond within a $600 \mathrm{~ms}$ interval $(500 \mathrm{~ms}$ of stimulus $+100 \mathrm{~ms}$ following it), the $N$ values, against the entire $N \times 500$ values in the $500 \mathrm{~ms}$ period preceding the stimulus. We corrected for the 600 multiple comparisons using false discovery rate (Benjamini and Yekutieli, 2001) with base $\alpha$ of 0.001 . Responses $<12 \mathrm{~ms}$ were excluded, and undetected intervals $<4$ ms that preceded and followed responses were categorized as misses and bridged with adjacent intervals. In REM sleep, where less data were available, we restricted analysis to those stimuli that had at least 15 trials.

Comparison across vigilance states. To compare the response of a unit to a stimulus across two states, we calculated the response magnitudes as follows: (1) We first identified time points in the response of each state, which were significantly above baseline activity (see above). (2) Next, we applied a "logical OR" between the two significant responsive periods, to produce a single vector that included all time points that were significant in any of the two states. (3) Then in each state separately, we averaged the firing rate across the time points in the combined vector, and (4) subtracted that state's baseline firing rate, to produce the state response magnitude.

To compare this response magnitude between pairs of states, we normalized the differences in response magnitudes by the maximum absolute value, to produce an unbiased gain factor as follows:

$$
\% \operatorname{Gain}_{(a-b)}=\frac{a-b}{\max (|a|,|b|)} \times 100
$$

where $a$ is the response magnitude in any of the sleep states and $b$ is the response magnitude in the wake state. Therefore, a negative gain factor indicates a reduced response during sleep, and vice versa. Distributions of gain factors across neurons are summarized as median, and [lower and upper] 95\% CIs around the median (Campbell and Gardner, 1988).

Latency of neuronal responses. To detect the peak latency of each neuronal cluster, we first quantified its responses to pure tones and click stimuli during wakefulness. For each stimulus, the peristimulus time histogram (PSTH) was smoothed with a Gaussian kernel ( $\sigma=8 \mathrm{~ms})$. Peak latency was first defined for each stimulus separately as the peak of a significant response within the first $100 \mathrm{~ms}$. Then, peak latency was defined for each neuron as the average of its peak latencies across stimuli. Computing response latencies jointly across all states did not reveal significant changes to the main results.

Definition of early-and late-responding neurons. Based on the result in Figure $6 B$, we identified two distinct populations of neurons that could be reliably separated according to their peak response latency, where $85 \%$ of neurons with responses $<20 \mathrm{~ms}$ were in AC and $89 \%$ of neurons with responses $>40 \mathrm{~ms}$ were in PRC. We therefore defined either early- or late-responding neurons as those with peak latency of $<20 \mathrm{~ms}$ or $>40 \mathrm{~ms}$ respectively, leaving out neurons with peak latencies of 20-40 ms from the analysis based on latency to avoid ambiguity. Precise cutoffs for early versus late neurons (e.g., 15/20/25 ms vs 30/40/50 ms) did not significantly affect the results.

To identify early- and late-responding neurons on the same microwires (see Fig. 6E,F), we focused on channels with at least two units exhibiting a difference $>20 \mathrm{~ms}$ in response latency, with the shorter latency $<20 \mathrm{~ms}$.

Neuronal ON/OFF periods. To detect ON/OFF periods, we first summed all (normalized) neuronal firing during NREM sleep periods (including light sleep) at millisecond resolution, for AC and PRC separately. Next, we smoothed the vectors with a Gaussian kernel ( $\sigma=50$ $\mathrm{ms})$, while excluding data immediately following $(<1000 \mathrm{~ms})$ auditory stimulation onset, to avoid false detections of auditory-induced reductions in firing as OFF periods. We further identified in the smoothed vectors periods above/below median activity, which lasted $>200 \mathrm{~ms}$, and defined the $30 \%$ of events with highest amplitude as ON/OFF periods, respectively. Identified ON/OFF periods that occurred $<200 \mathrm{~ms}$ before auditory trial onsets were discarded. Trials that fell during such periods, or in periods not categorized as ON or OFF, were not included in further analysis. We converged on these parameters after extensive visual inspection of detected ON/OFF periods in data of each animal. Together, $15 \pm$ $2.3 \%$ of NREM trials occurred during either ON or OFF periods and were included in this analysis. The effect of regional ON/OFF periods on auditory responses was assessed by the gain calculation above (as when comparing two states, but this time comparing ON vs OFF within NREM sleep), with a minimum of 8 trials in each condition (to allow reliable comparisons).

Statistics. All data analysis was performed using custom MATLAB scripts. Unless stated otherwise, a two-tailed Mann-Whitney $U$ test was used to assess statistical significance. Multiple comparisons were corrected via false discovery rate (Benjamini and Yekutieli, 2001). Stimuli were presented in a pseudorandom order and interleaved across vigilance states due to the natural fragmented sleep of rodents. Sleep scoring was blind to the timing of auditory trials.

Data and code availability. The data that support the findings of this study and the computer code used to generate the results are available from the corresponding author upon reasonable request. 
A

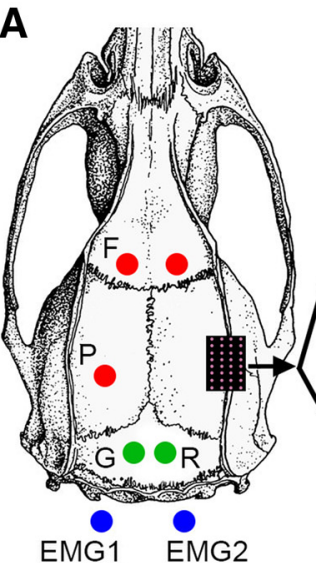

D

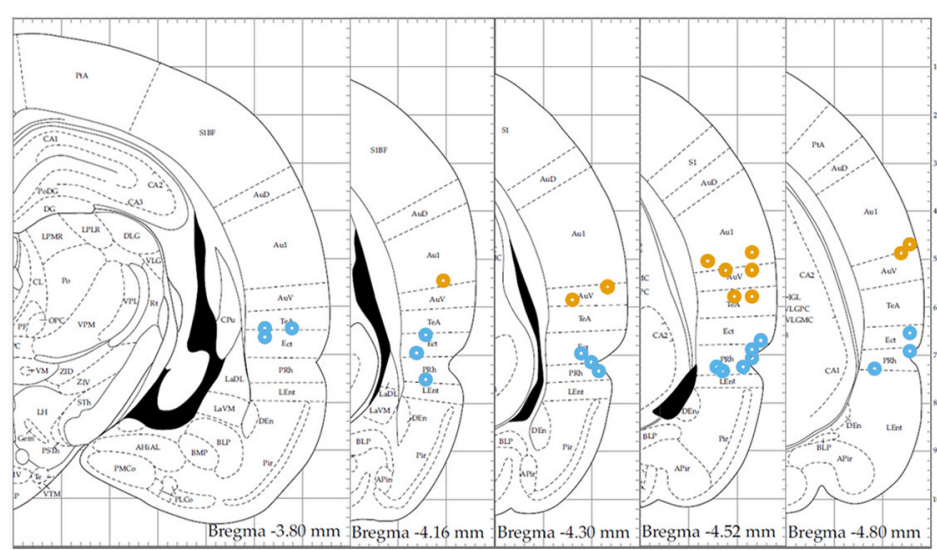

B

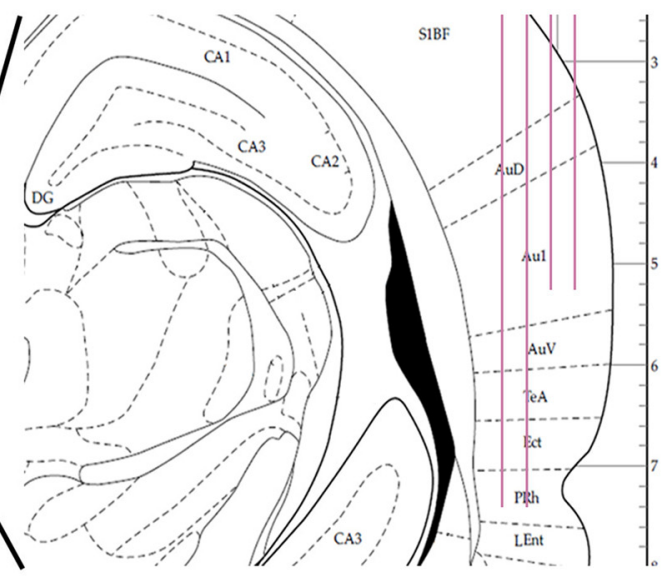

E


Figure 1. Experimental design. $\boldsymbol{A}$, Surgical plan, including frontal (F) and parietal (P) EEG screws (red circles), ground (G) and reference (R) screws (green circles) above cerebellum, and two wires (blue circles) recording EMG from neck muscles. Microwire arrays were implanted in rat temporal lobe through a craniotomy (black rectangle with purple dots marking each wire). $\boldsymbol{B}$, Coronal section $(-4.16 \mathrm{~mm}$ from bregma) of the implantation area, showing the implantation plan of 32-channel microwire arrays ( 4 rows $\times 8$ wires each) targeting AC ( 2 shorter rows) and PRC ( 2 longer rows). C, Representative histological verification of electrode positions. Red dashed lines indicate estimated area borders. Red arrows point to detectable electrode tips in AC and PRC. Inset, Abbreviations of brain regions. PRC (yellow font) includes both area 36 (ectorhinal cortex) (Kealy and Commins, 2011) and area 35 (PRh). D, Five coronal sections (spanning -3.80 to -4.80 from bregma) showing the locations of electrodes identified in histology (orange/blue circles for AC/PRC microwires). $E$, Experimental setup diagram: rats were placed individually in a sound-attenuating chamber, while auditory stimuli were delivered free-field from a speaker mounted $55 \mathrm{~cm}$ above the cage center. Intracranial electrophysiology and sleep polysomnography (EEG, EMG, video) were continuously collected while rats were behaving and sleeping freely.

\section{Results}

To study sensory processing along the auditory-mnemonic hierarchy across wakefulness and sleep, we implanted adult male rats $(n=10)$ with microwires targeting the right $\mathrm{AC}(n=7)$ and/or PRC ( $n=7$; Fig. 1$)$. After a week of recovery and habituation, we presented a wide battery of sounds (including tones, clicks and click trains, white noise, rat vocalizations, and vocoded vocalizations) in 12 experimental sessions lasting $5-8 \mathrm{~h}$ each, as animals spontaneously switched between vigilance states in their home cage during the light phase.

Sleep scoring was performed based on EEG, EMG, and video (Fig. 2A; see Materials and Methods). Quiet wake, NREM sleep, and REM sleep composed $15 \pm 6.3 \%, 41 \pm 6.2 \%$, and $8 \pm 2.4 \%$ of experiment time, respectively (mean $\pm \mathrm{SD}$; Fig. $2 B, C$ ) and exhibited characteristic changes in EEG power spectra, including increased slow-wave activity in NREM sleep, and higher theta/ high-frequency activity in wakefulness and REM sleep (Fig. 2D).

We isolated 435 neuronal clusters (130 single units, 305 multiunit clusters; Fig. $3 A-C$ ) that significantly responded to auditory stimuli in at least one vigilance state, of 485 neuronal clusters in total (9\% and $14 \%$ of clusters in AC and PRC, respectively, did not show significant responses to any presented sound). To compare between activities in the two regions, responsive neurons were sep- arated into AC $(n=178)$ and PRC $(n=132)$ based on histology in all cases where that provided unequivocal information $(n=8$ sessions in 6 rats) (Fig. 1). Analysis of baseline firing rates revealed that AC neurons exhibited $21 \%-26 \%$ higher firing rates during wakefulness $(6.0 \pm 0.6 \mathrm{~Hz})$ or REM sleep $(5.6 \pm 0.6 \mathrm{~Hz})$ compared with NREM sleep $\left(4.4 \pm 0.4 \mathrm{~Hz}\right.$, $p$ values $\leq 10^{-8}$ via signed-rank tests). In contrast, baseline firing rates of PRC neurons was higher during REM sleep $(5.3 \pm 0.6 \mathrm{~Hz})$ compared with wakefulness $(4.7 \pm 0.5 \mathrm{~Hz}$, $12 \%$ difference) and NREM sleep ( $4.3 \pm 0.4 \mathrm{~Hz}, 23 \%$ difference; $p$ values $\leq 0.001$, via signed-rank tests; Fig. $3 D$ ).

\section{Auditory responses in AC and PRC neurons across wakefulness and sleep}

To examine auditory-evoked discharges, neuronal responses were aligned to sound onset, and an automatic algorithm detected significantly responsive intervals around stimulus presentation (Fig. 3E; see Materials and Methods). We compared auditory spiking responses across vigilance states in each cortical region. In line with previous studies (Issa and Wang, 2008; Nir et al., 2015), spiking responses in AC neurons exhibited similar profiles across vigilance states, whereas PRC neurons exhibited weaker responses in both NREM and REM sleep (Fig. 4). 
A

Active Wake

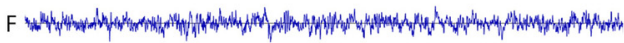

$P$ H.

$\sum_{\Psi}^{\cup}$

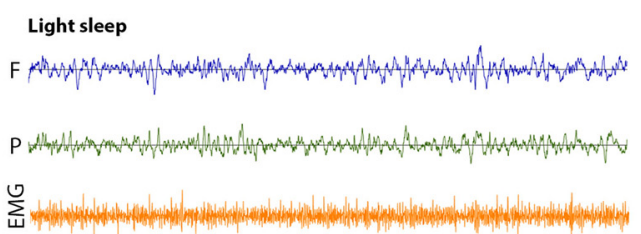

NREM-REM transition

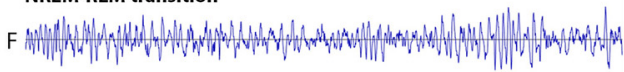

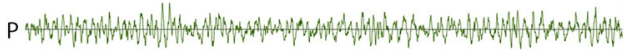

$\sum$

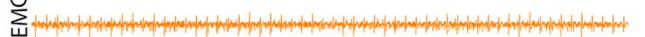

\section{Quiet Wake}

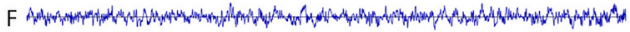

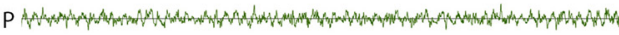

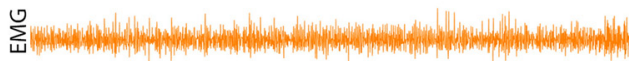

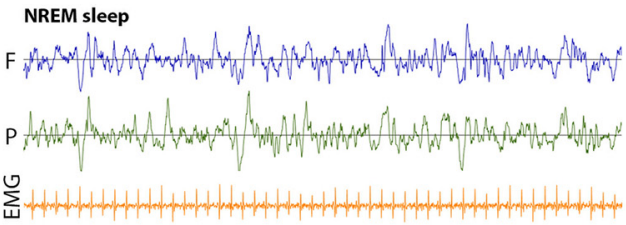

REM sleep

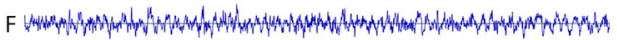

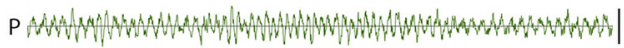

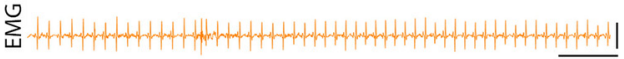

B
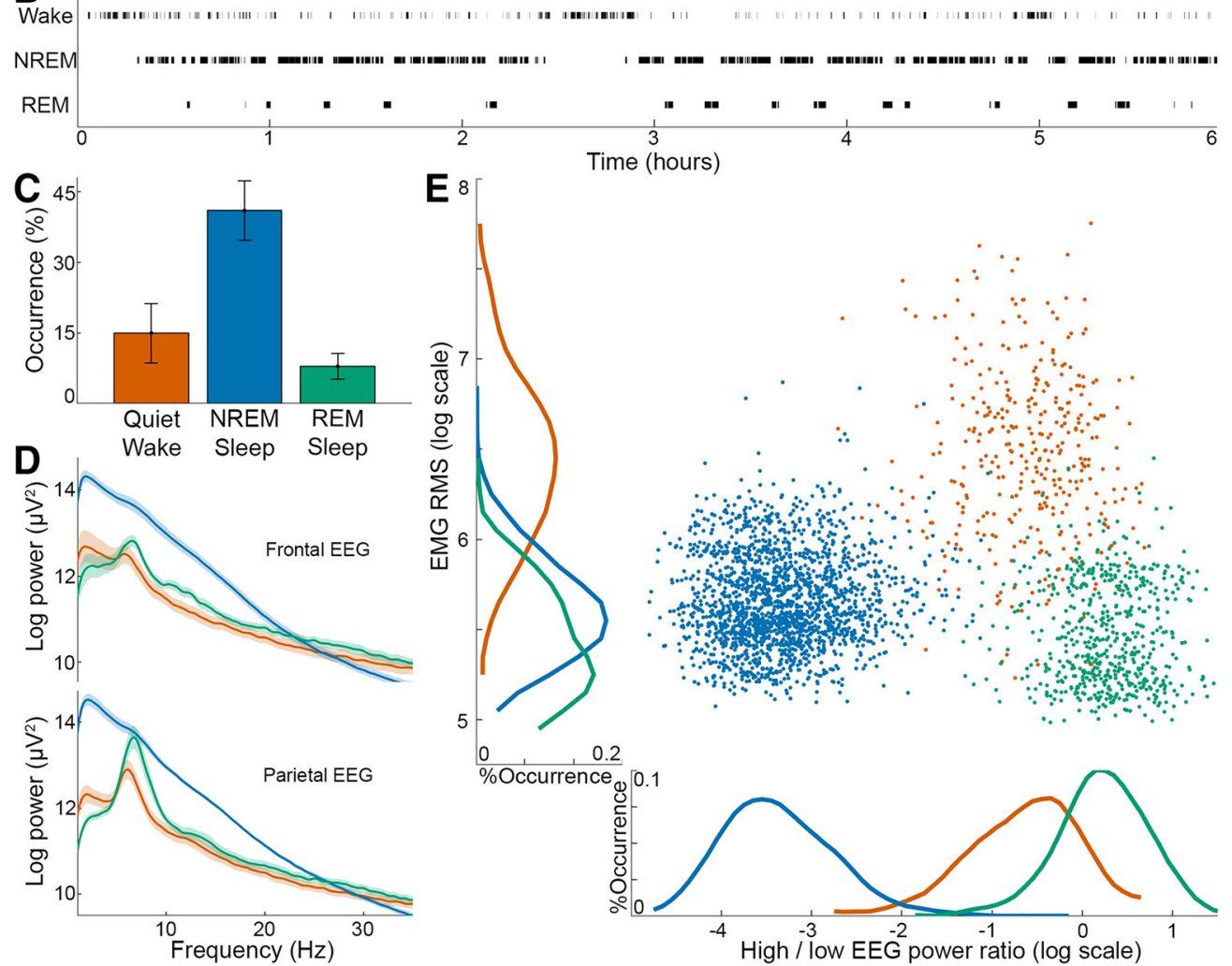

Figure 2. Sleep scoring. A, Representative polygraphic traces of the different vigilance states. In each panel, EEG above the frontal (blue) and parietal (green) cortices is shown above the EMG (orange). Horizontal line at bottom right corner indicates 1 s time scale. Vertical lines indicate $1 \mathrm{mV}$ and $0.25 \mathrm{mV}$ for EEGs and EMG, respectively. $\boldsymbol{B}$, Hypnogram showing the time course of vigilance state transitions in a representative experiment. Black ticks represent the states used for full analysis (top row, quiet wake; middle row, NREM sleep; bottom row, REM sleep). $C$, Occurrence (percent of time, $y$ axis) of vigilance states ( $x$ axis) during experiments. Error bars indicate $S D$ across sessions. Colors in all panels represent vigilance states: red represents quiet wake; dark blue represents NREM sleep; green represents REM sleep. D, EEG spectral power across vigilance states in frontal EEG (top) and parietal EEG (bottom). Thick lines and shading represent mean \pm SEM across experimental sessions. $\boldsymbol{E}$, A representative scatter plot distribution of EMG root mean square (y axis) versus frontal EEG power distribution (ratio between power in high [ $>25 \mathrm{~Hz}]$ versus low [ $<5$ $\mathrm{Hz}$ ] frequencies, $x$ axis) in each $4 \mathrm{~s}$ epoch. Both sleep states have low EMG, whereas EEG is dominated by high-frequency activity in wake and REM sleep.

We proceeded to systematically and quantitatively compare the response magnitude of each neuron in wakefulness and sleep states across the entire dataset (Fig. 5A). PRC neurons showed attenuation with median gain factor of $-47 \%(\mathrm{CI}=[-56 \%$, $-34 \%])$ in NREM sleep and $-36 \%(\mathrm{CI}=[-43 \%,-30 \%])$ in
REM sleep (Fig. 5B). By contrast, AC neurons only exhibited modest changes, with median gain factor of $-12 \%(\mathrm{CI}=$ $[-20 \%,-3 \%])$, and $-6 \%(\mathrm{CI}=[-19 \%, 2 \%])$ in NREM sleep and REM sleep, respectively. When quantifying the fraction of responsive units that either decreased or increased their driven 
A
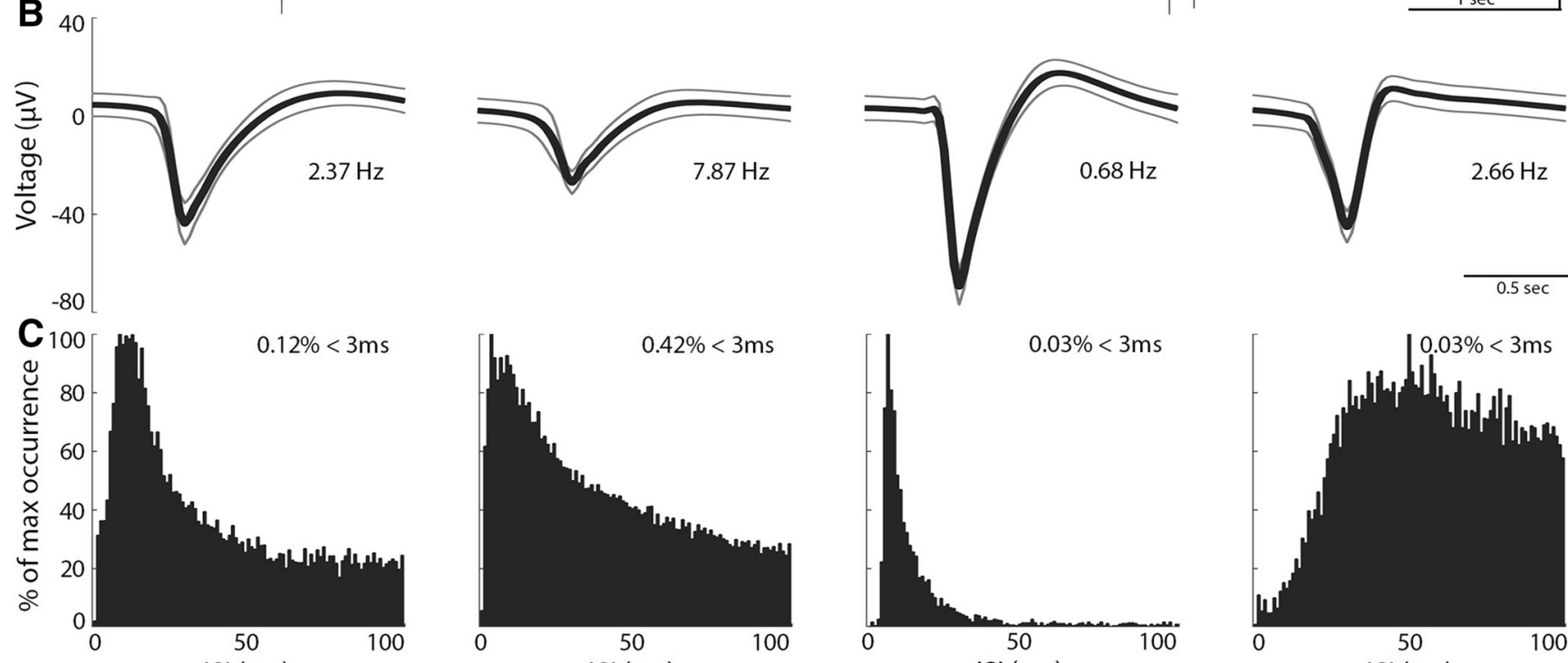

ISI (ms)



ISI (ms)

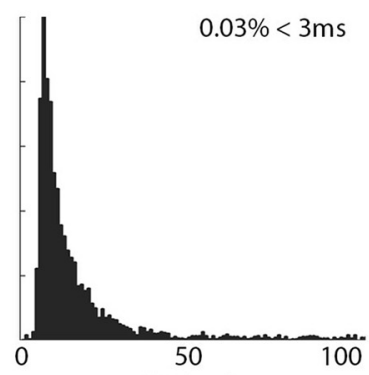

ISI (ms)

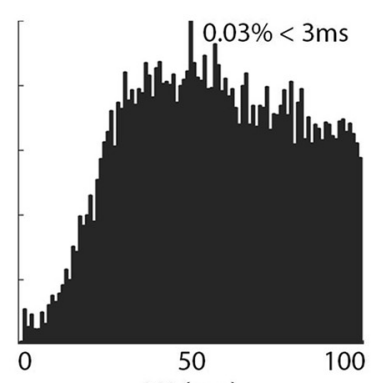

ISI (ms)
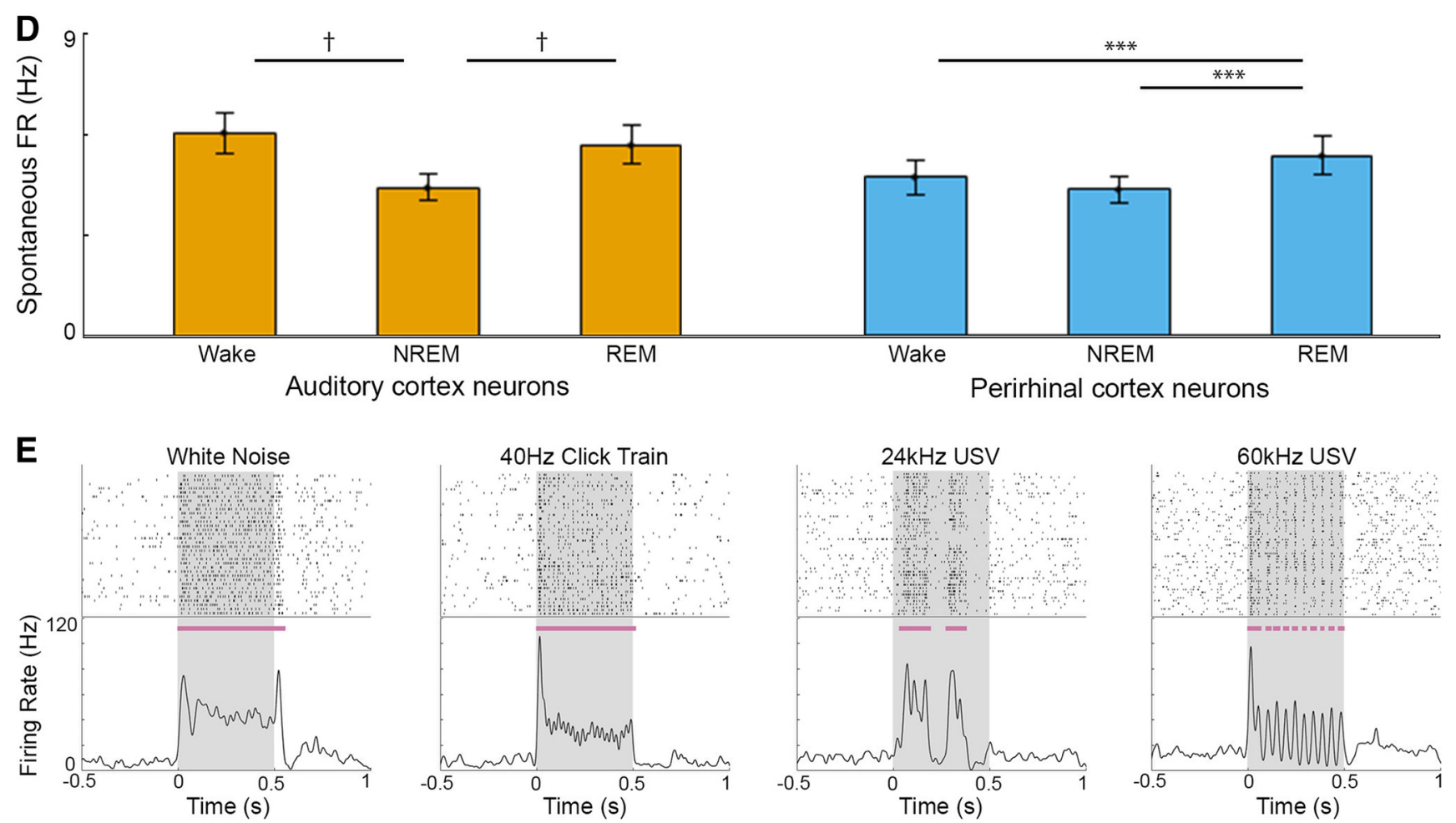

Figure 3. Neuronal spiking activity in AC and PRC. $A$, Representative 10 s trace of high-pass filtered ( $300-5000 \mathrm{~Hz}$ ) local field potential used for identification of spiking activity. Red line indicates the automatic spike detection threshold. $\boldsymbol{B}$, AP waveforms of three detected single units and one multiunit cluster (second from left) in the above channel. Thick lines and thinner frames represent mean \pm SD. Numbers indicate the mean firing rate of each unit. C, Corresponding interspike interval (ISI) histograms of each unit. Percentage in top right corner indicates the prevalence of APs occurring successively within $0-3 \mathrm{~ms}$. D, Baseline firing rate across vigilance states in AC (left, orange, $n=178$ ) and PRC (right, blue, $n=132$ ) neurons. Error bars indicate SEM across neurons. ${ }^{* * *} p=0.001 ; \dagger p=10^{-8}$; signed-rank test. $\boldsymbol{E}$, Representative raster plots (top) and PSTHs (bottom) of neuronal responses. Gray shading represents stimulus presentation times. Horizontal purple bars represent automatically detected response intervals (see Materials and Methods). USV, Ultrasonic vocalization. 

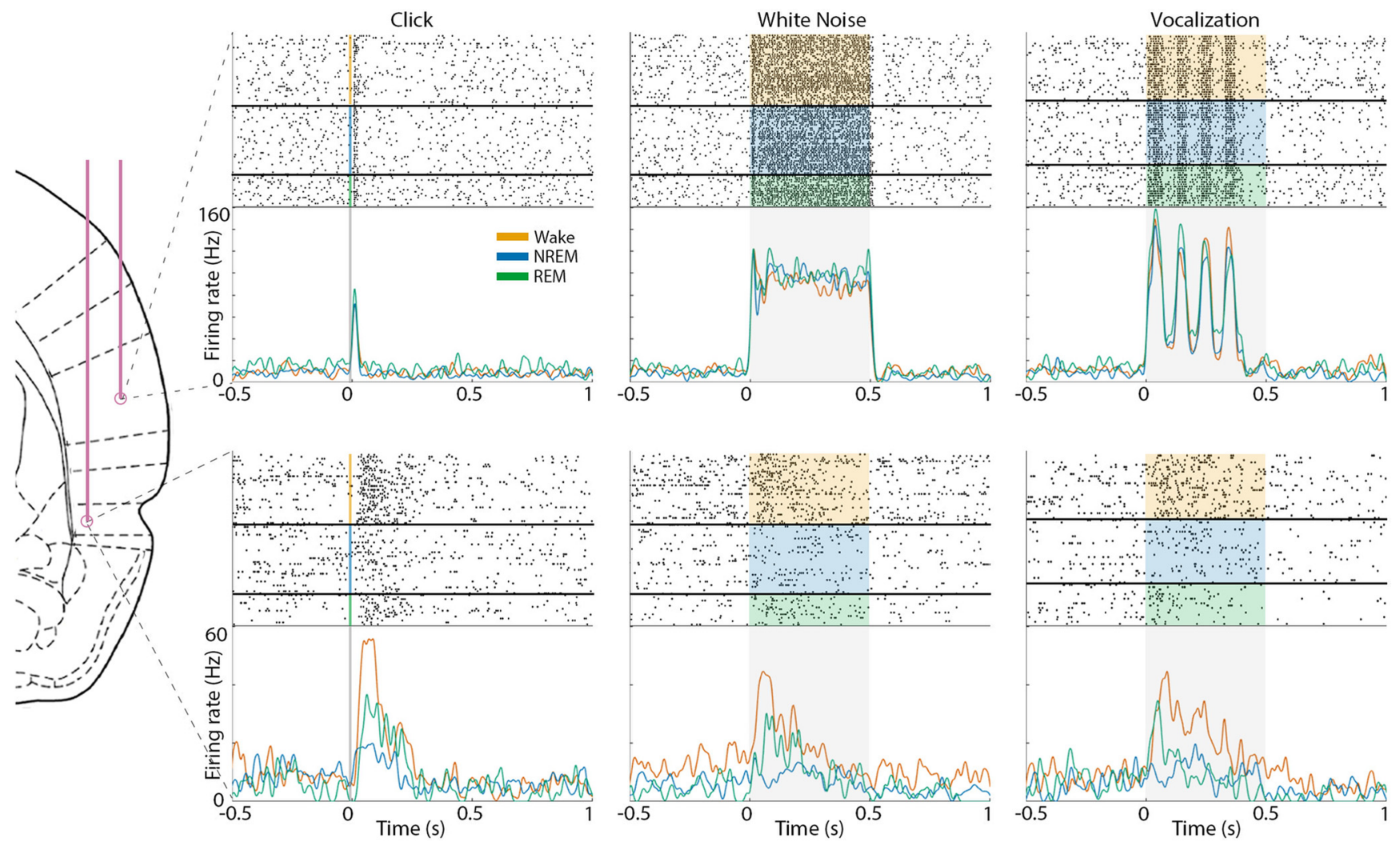

Figure 4. Example auditory responses in AC and PRC neurons across wakefulness and sleep. Raster plots (top panels) and PSTH (bottom panels) show representative auditory responses in an AC neuron (top row) and a PRC neuron (bottom row) recorded simultaneously. Columns show responses to different stimuli, including a click (left), $12 \mathrm{kHz}$ white noise burst (middle), and $66 \mathrm{kHz}$ ultrasonic vocalization (right). In each panel, shaded area represents stimulus duration. Raster plots are grouped by vigilance state: orange represents wake; blue represents NREM sleep; green represents REM sleep. PSTH traces of different vigilance states are superimposed (colors as above).

firing rate during sleep, we only found modest differences in AC (59/56\% of units decreased their responses and $41 / 44 \%$ increased their responses in NREM/REM sleep, respectively), whereas PRC units predominantly decreases their responses in sleep $(80 / 77 \%$ of units decreased their responses and 20/23\% increased their responses in NREM/REM sleep, respectively). The distribution of PRC sleep attenuation gain factors was significantly more negative than that of $\mathrm{AC}\left(p=10^{-5}\right.$ and $p=10^{-4}$ via Mann-Whitney test for NREM sleep and REM sleep, respectively). Results were largely comparable when gain factors were computed for each stimulus separately (median gain factor in AC: $3 \%$ and $-11 \%$ during NREM and REM sleep, respectively; in PRC: $-41 \%$ and $-37 \%$ during NREM and REM sleep, respectively). Analyzing the effects of sleep separately for different sound categories (i.e., pure tones, white noise, clicks, vocalizations) did not reveal significant differences between stimulus categories. Separate analysis of light NREM sleep epochs (see Materials and Methods) showed a median gain modulation of $-26 \%(\mathrm{CI}=[-37 \%$, $-17 \%]$; data not shown). All results presented here as gain factor of raw firing rates (spikes/s); however, similar results are obtained when comparing responses when expressed as $z$ scores relative to baseline.

\section{Response latency of each neuronal cluster is associated with its sleep attenuation}

Auditory responsive neuronal clusters fell into two distinct categories based on their peak latency during wakefulness (Fig. 6A). Responses of early-responding neurons with peak latencies $<20$ ms were mostly (85\%) observed in AC, whereas the majority $(89 \%)$ of late-responding neurons with peak latencies $>40 \mathrm{~ms}$ were found in PRC (Fig. 6B; see Materials and Methods). There was also a correlation $(r=-0.24, p=0.001)$ between response latency and the width of AP wave, which manifested as a significant $\left(p=1.4 \times 10^{-4}\right)$ tendency for narrow AP waveforms (median width $=0.11 \mathrm{~ms}$ ) in short latency neurons compared with long latency units (median AP width $=0.15 \mathrm{~ms}$ ). Next, we went beyond a binary separation to early- and late-responding neurons and found a significant correlation $\left(r=-0.55, p=10^{-15}\right)$ between peak latency and NREM sleep gain attenuation (Fig. $6 C)$, and a more modest correlation $(r=-0.24, p=0.001)$ between peak latency and REM sleep gain attenuation (data not shown).

Given the tight link between response latency and NREM sleep attenuation, we reexamined how sleep affects the auditory responses of "atypical" neurons $(n=41)$ where latency did not match the typical latency profile of the anatomical region (i.e., late-responding AC neurons or early-responding PRC neurons). We found that late-responding AC neurons show a substantial attenuation profile resembling that observed in PRC (gain factor of $-43 \%, \mathrm{CI}=[-60 \%,-25 \%])$, whereas early-responding PRC neurons only showed modest attenuation in NREM sleep, as was observed in $\mathrm{AC}$ (gain factor of $-3 \%, \mathrm{CI}=[-16 \%, 33 \%]$; Fig. $6 D)$. Finally, in the few cases where two neuronal clusters were identified on the same microwire and showed distinct response latencies ( $n=15$ pairs; Fig. $6 E$ ), we found a robust ( 8 -fold) difference in gain attenuation during NREM sleep such that earlyresponding neurons showed a modest average gain $(-5.4 \pm$ $9.8 \%)$ versus robust $(-41 \pm 8.6 \%)$ attenuation for lateresponding neurons (mean \pm SEM, $p=0.003$ via signed-rank test; Fig. $6 F$ ). In REM sleep, attenuation did not clearly follow 
response latency as was the case during NREM sleep (data not shown), nor did we observe a significant difference between attenuation for neuronal pairs with different latencies recorded on same microelectrodes $(p=0.07,5 \%$ in earlyresponding neurons vs $-20 \%$ in lateresponding neurons).

Finally, we compared the effect of regional ON/OFF periods on auditory responses during NREM sleep. We observed a complex profile where early-responding neurons showed modestly stronger responses for trials occurring during $\mathrm{ON}$ periods $($ median ON/OFF gain $=12 \%$, $\mathrm{CI}=[-1 \%, 18 \%], p=0.52)$, whereas late-responding neurons showed significantly stronger responses when stimuli were delivered during local OFF periods (median ON/OFF gain $=-41 \%, \mathrm{CI}=$ $\left.[-44 \%,-19 \%], p=10^{-4}\right)$.

\section{Discussion}

The present results reveal how natural sleep differentially affects auditory responses along the cortical sensory hierarchy. We find that PRC exhibits robust attenuation of response magnitude in both NREM sleep and REM sleep, whereas AC neurons are only modestly affected by sleep. Moreover, response attenuation is correlated with response latency in individual neurons. Dividing neurons according to their response latency can explain the NREM sleep attenuation profiles based on cortical region (AC vs PRC), and can also go beyond anatomical parcellation in showing modest sleep attenuation in early-responding PRC neurons versus robust attenuation in late-responding AC neurons. Given the preserved auditory responses in previous studies, our results provide the first direct neuronal evidence for attenuation of auditory responses in natural sleep, and pave the road for future studies to dissect the mediating mechanisms.

Downstream to the primary AC, a previous study in marmosets (Issa and Wang, 2008) showed that secondary auditory cortices also exhibit preserved responses between vigilance states. However, studies of sensory processing beyond early sensory regions during natural sleep are limited. One study (Vinnik et al., 2012) found that a subset of hippocampal neurons in the rat do exhibit robust sensory responses during natural sleep. Another study (Pereira et al., 2007) reported increased hippocampal responses to microsimulation of the rodent somatosensory whisker system during sleep. This discrepancy with the robust attenuation we find here in rat PRC may reflect differences in sensory modalities, or differences between natural stimuli used here versus ultra-short electrical stimulation.

In AC, comparable early responses in natural sleep observed here are in line with several recent reports (Peña et al., 1999; Edeline et al., 2001; Issa and Wang, 2008; Nir et al., 2015). In addition, a recent study comparing auditory responses at the neuronal level in humans across wakefulness and light anesthesia
NREM sleep
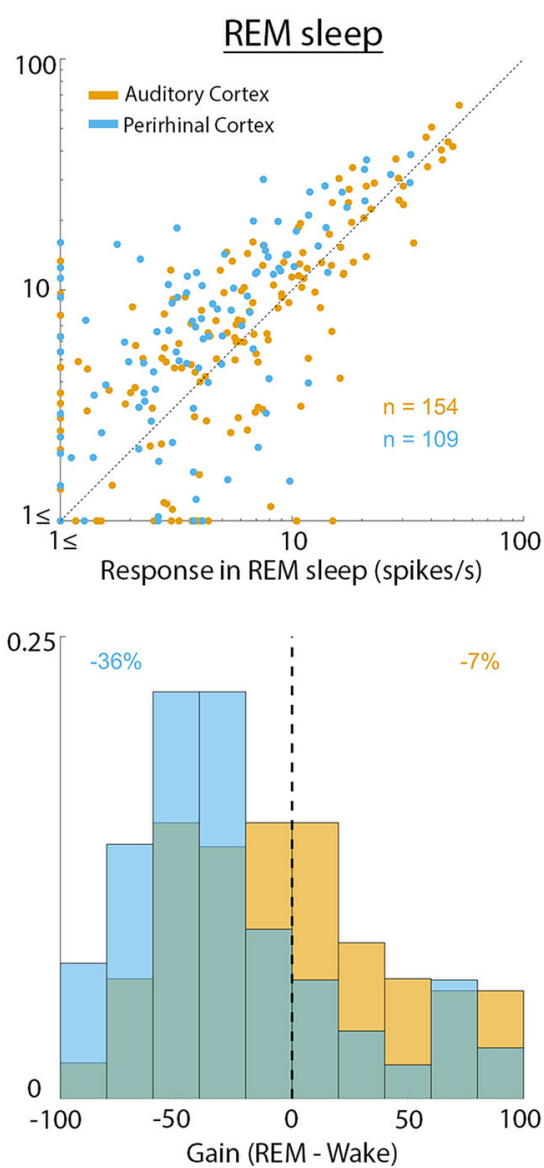

Figure 5. Quantitative comparison of auditory responses in AC and PRC neurons across wakefulness and sleep. $\boldsymbol{A}$, Scatter plot of wakefulness). $\boldsymbol{B}$, Distribution of gain factors (see Materials and Methods) for AC neurons versus PRC neurons. Colors are as in $\boldsymbol{A}$. Vertical dashed line indicates zero gain. Numbers in top corners indicate the median gain factor in each neuronal population.

found a similar distinction between relatively preserved early responses in AC versus robust attenuation of late responses in association cortex (Krom et al., 2018).

What could be the source of apparent discrepancy with earlier studies in other sensory modalities that reported attenuated spiking responses in primary cortices during natural sleep? One possibility is that auditory processing is relatively immune to thalamic gating. Along this line, residual sensory processing during sleep has been demonstrated mainly using acoustic and linguistic stimuli (Bastuji and García-Larrea, 1999; Hennevin et al., 2007). However, a closer examination of the original results (Evarts, 1963) indicates that the response latency of attenuated responses is $\sim 80-100 \mathrm{~ms}$, in line with our observation that, during NREM sleep, response latency better predicts sleep attenuation than anatomical region (Fig. 6). Other classical studies in primary somatosensory and visual cortices (Gücer, 1979; Livingstone and Hubel, 1981) only considered neurons with robust responses during wakefulness and quantified their response profiles in sleep, leaving out potential responses that could be stronger/exclusively present in sleep. Future studies comparing responses between vigilance states should take into consideration the existence of sleep responses that diminish during wakefulness.

We find that early AC responses are comparable across both NREM and REM sleep, whereas PRC responses were attenuated in NREM sleep, and to a lesser extent during REM sleep. We 
A
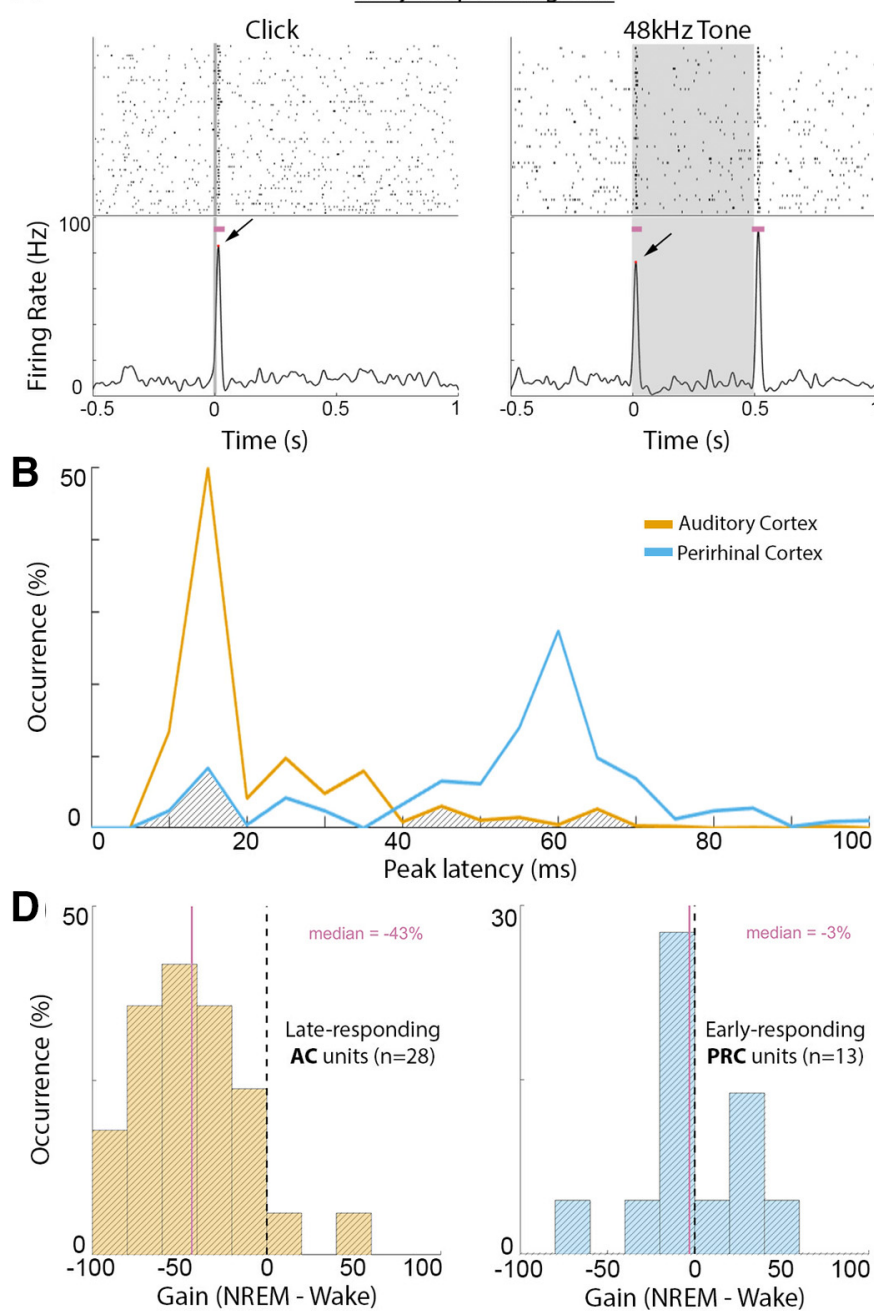

$\mathbf{E}$

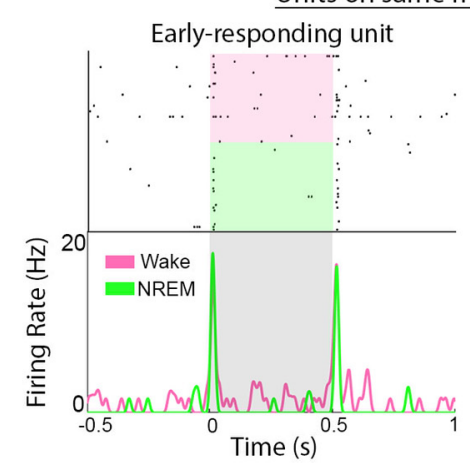

Units on same microwire electrode

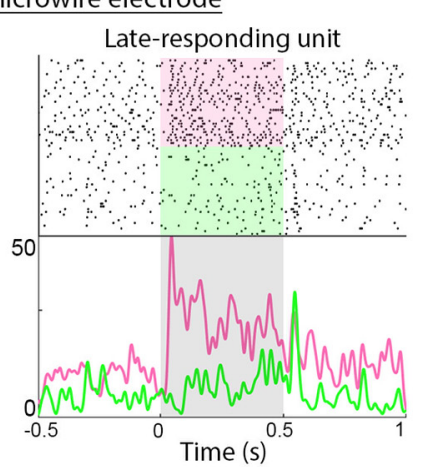

$48 \mathrm{kHzTone}$

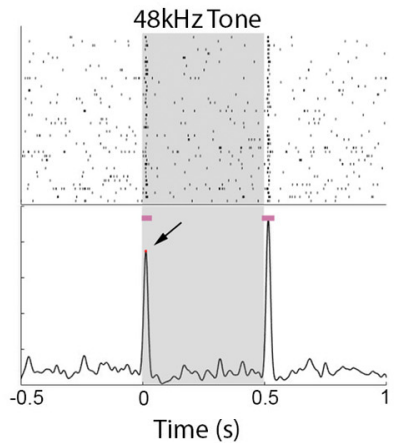

(1)
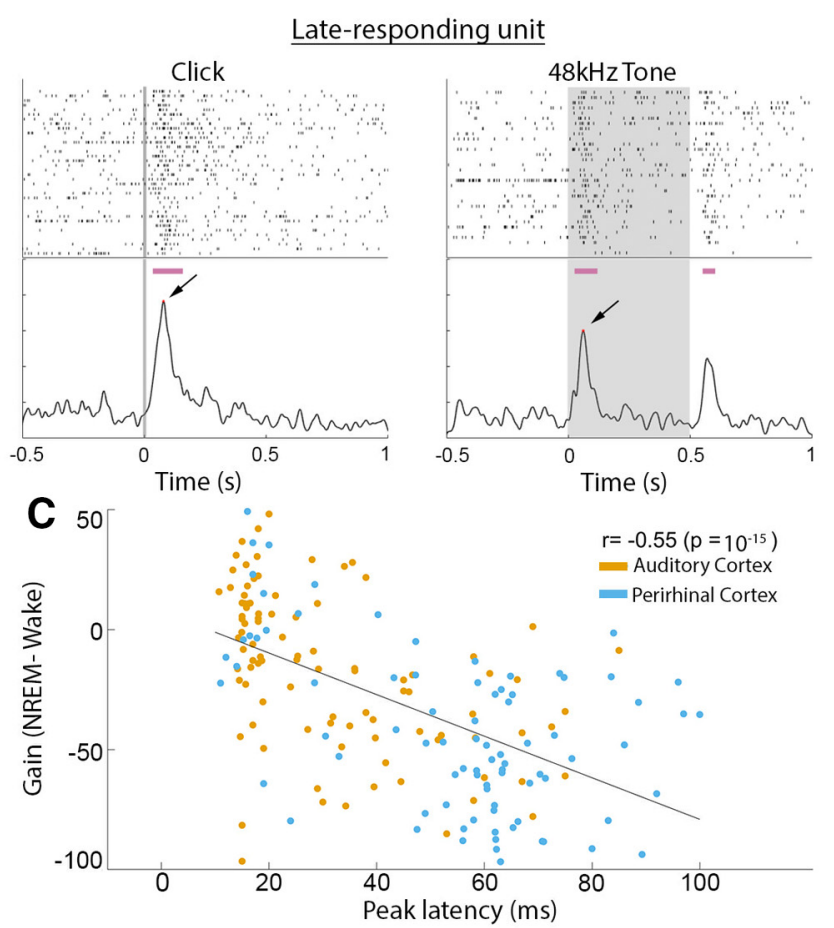

$\mathbf{F}$

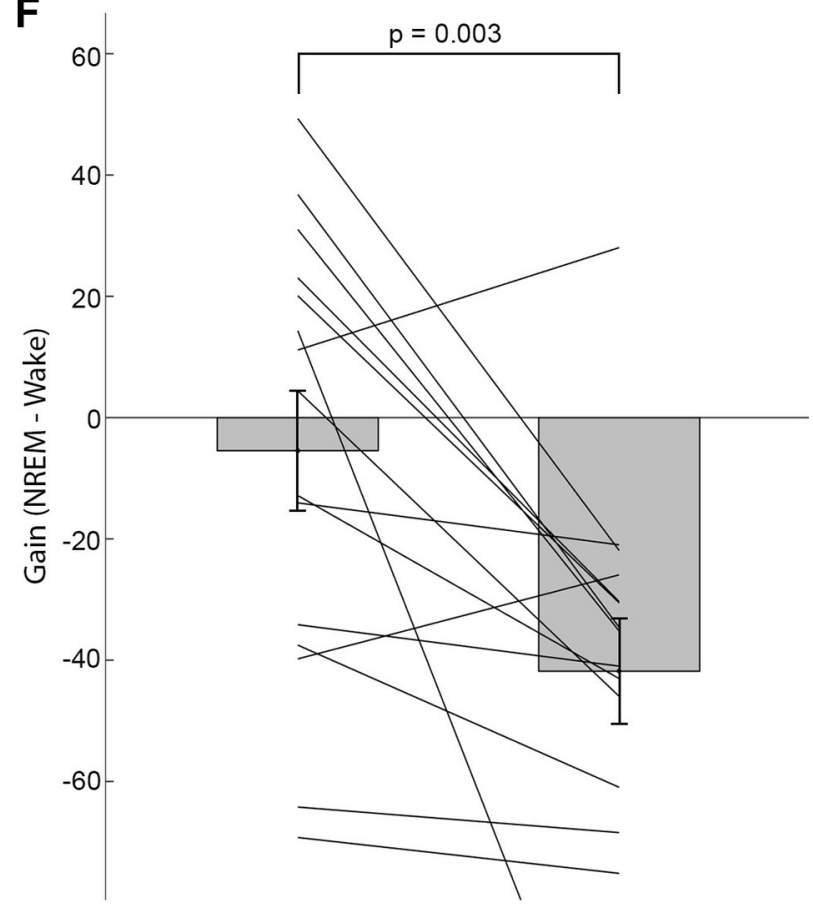

Early-responding units

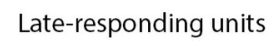

Figure 6. Response latency better predicts attenuation than anatomical region does during NREM sleep. $\boldsymbol{A}$, Example responses to click and tone pip stimuli during wakefulness in an earlyresponding neuron (two left columns) versus a late-responding neuron (two right columns). Gray shades represent stimulus presentation times. Horizontal purple bars represent automatically detected response intervals. Arrows indicate peak response used for early versus late categorization (latencies from left to right: $16,14,80$, and 62 ms). $\boldsymbol{B}$, Distribution of peak response latencies in $\mathrm{AC}$ (orange line, typically $<20 \mathrm{~ms}$ ) versus PRC (blue line, typically $>40 \mathrm{~ms}$ ). Gray shading represents clusters with "atypical" latencies in each area ( $>40 \mathrm{~ms}$ in $\mathrm{AC}$ and $<20 \mathrm{~ms}$ in PRC) that are analyzed separately in $\boldsymbol{D}$. C, Scatter plot of wake versus NREM sleep gain factor ( $y$ axis) versus peak response latency ( $x$ axis) for each neuron, colored accordance to anatomical region. Top right corner represents Pearson correlation coefficients and corresponding statistical significance, representing a tendency of late-responding neurons to exhibit larger differences in response magnitude between wakefulness and NREM sleep. $\boldsymbol{D}$, Examination of neurons with "atypical" latencies for their region (B, gray shaded areas). Left, Wake/NREM sleep gain factor distribution of AC neurons with long ( $>40 \mathrm{~ms}$ ) response latency $(n=28)$. Right, Wake/NREM sleep gain factor distribution of PRC neurons with short $(<20 \mathrm{~ms})$ response latency ( $n=13)$. Vertical purple lines indicate median gain factors (also in purple text on top). Latency better predicts sleep attenuation than anatomical region does. E, Example responses (raster plot and PSTH) of two neuronal clusters identified on the same microelectrode, showing different peak latencies (left, $14 \mathrm{~ms}$; right, $45 \mathrm{~ms}$ ) in response to the same sound ( $3 \mathrm{kHz}$ pure tone). Shading represents stimulus duration. Pink and green in raster plot and in PSTH represent trials during wakefulness and NREM sleep, respectively. $\boldsymbol{F}$, Sleep attenuation gain factors in 15 pairs of early-responding neurons (left, mean -5.4 ) and late-responding neurons (right, mean -41 ) identified on the same microelectrodes. $p=0.003$ (signed-rank test). Bars and error bars indicate mean and SEM, respectively. Lines connect gain factors in pairs of neurons recorded on the same microelectrodes. 
propose that the degree of response attenuation in high-order cortex mirrors the depth of sleep as defined behaviorally. Indeed, arousal thresholds in the rat are higher in NREM sleep (compared with REM sleep) and with increased slow-wave activity (Neckelmann and Ursin, 1993; Hayat et al., 2019). Along this line, light NREM sleep is associated with a lower arousal threshold (Ermis et al., 2010; Hayat et al., 2019), and we find it exhibits a more modest response attenuation than both NREM sleep and REM sleep. Such reductions in neuronal responsiveness and arousal threshold have been suggested to be related to changes in neuromodulatory milieu (Cho et al., 2017; Hayat et al., 2019), which may affect processing characteristics of different areas as the PRC (Zerbi et al., 2019).

The robust correlation of NREM sleep attenuation with response latency suggests that NREM sleep may exert a gradual attenuation of cortical responses, rather than imposing a discrete "gate" at a specific brain region. As a first approximation, AC and PRC are associated with different response latency profiles (Lee et al., 2016) and differ also in their degree of response attenuation during sleep. However, a closer examination of neuronal clusters with "atypical" latencies reveals that their attenuation during NREM sleep is better predicted by their classification to early/late responders rather than their anatomical location (Fig. 6). The fact that late responses exhibit stronger attenuation in NREM sleep is in line with the notion of breakdown of cortical effective connectivity during NREM sleep (Massimini et al., 2005) that may lead to greater attenuation with increasing synaptic connections. By contrast, we could not reveal robust relation between response latency and attenuation in REM sleep, in line with the partial recovery of effective connectivity during REM sleep (Massimini et al., 2010). Thus, during REM sleep, other mechanisms may be at play. For example, although speculative, it is possible that, during REM sleep, ongoing mnemonic activity during dreaming interferes with bottom-up sensory processes (Nir and Tononi, 2010; Andrillon et al., 2016).

Of note, we used a passive auditory stimulation scheme where sounds were not associated with specific behaviors or rewards. This approach allowed us to distill changes related to vigilance state per se, without confounding processes that boost responses to behaviorally relevant stimuli during wakefulness. Future studies could compare how sleep may differentially affect responses to such salient stimuli beyond the effects described here.

In conclusion, we find a robust attenuation of late responses in high-order sensory association cortex during natural sleep at the level of individual neuronal spiking. Building on the anatomical and temporal markers of sleep attenuation found here, future studies can elucidate the precise mechanisms underlying the sensory disconnection of sleep.

\section{References}

Allen TA, Furtak SC, Brown TH (2007) Single-unit responses to $22 \mathrm{kHz}$ ultrasonic vocalizations in rat perirhinal cortex. Behav Brain Res 182:327336.

Andrillon T, Poulsen AT, Hansen LK, Léger D, Kouider S (2016) Neural markers of responsiveness to the environment in human sleep. J Neurosci 36:6583-6596.

Bastuji H, García-Larrea L (1999) Evoked potentials as a tool for the investigation of human sleep. Sleep Med Rev 3:23-45.

Benjamini Y, Yekutieli D (2001) The control of the false discovery rate in multiple testing under dependency. Ann Stat 29:1165-1188.

Campbell MJ, Gardner MJ (1988) Statistics in medicine: calculating confidence intervals for some non-parametric analyses. Br Med J 296:14541456.

Carskadon MA, Dement WC (2011) Normal human sleep: an overview. In:
Principles and practice of sleep medicine, Ed 5 (Kryger MH, Roth T, Dement WC, eds), pp 16-26. St. Louis: Saunders/Elsevier.

Cho JR, Treweek JB, Robinson JE, Xiao C, Bremner LR, Greenbaum A, Gradinaru V (2017) Dorsal raphe dopamine neurons modulate arousal and promote wakefulness by salient stimuli. Neuron 94:1205-1219.e8.

Cirelli C, Tononi G (2008) Is sleep essential? PLoS Biol 6:e216.

Datta S, Hobson JA (2000) The rat as an experimental model for sleep neurophysiology. Behav Neurosci 114:1239-1244.

de Curtis M, Paré D (2004) The rhinal cortices: a wall of inhibition between the neocortex and the hippocampus. Prog Neurobiol 74:101-110.

Doron NN, Ledoux JE, Semple MN (2002) Redefining the tonotopic core of rat auditory cortex: physiological evidence for a posterior field. J Comp Neurol 453:345-360.

Edeline JM, Dutrieux G, Manunta Y, Hennevin E (2001) Diversity of receptive field changes in auditory cortex during natural sleep. Eur J Neurosci 14:1865-1880.

Ermis U, Krakow K, Voss U (2010) Arousal thresholds during human tonic and phasic REM sleep. J Sleep Res 19:400-406.

Evarts EV (1963) Photically evoked responses in visual cortex units during sleep and waking. J Neurophysiol Publ 26:229-248.

Furtak SC, Allen TA, Brown TH (2007) Single-unit firing in rat perirhinal cortex caused by fear conditioning to arbitrary and ecological stimuli. J Neurosci 27:12277-12291.

Gücer G (1979) The effect of sleep upon the transmission of afferent activity in the somatic afferent system. Exp Brain Res 34:287-298.

Hayat H, Regev N, Matosevich N, Sales A, Paredes-Rodriguez E, Krom AJ, Bergman L, Li Y, Lavigne M, Kremer EJ, Yizhar O, Pickering AE, Nir Y (2019) Locus-coeruleus norepinephrine activity gates sensory-evoked awakenings from sleep. bioRxiv. doi: 10.1101/539502.

Hennevin E, Huetz C, Edeline JM (2007) Neural representations during sleep: from sensory processing to memory traces. Neurobiol Learn Mem 87:416-440.

Issa EB, Wang X (2008) Sensory responses during sleep in primate primary and secondary auditory cortex. J Neurosci 28:14467-14480.

Kakigi R, Naka D, Okusa T, Wang X, Inui K, Qiu Y, Tran TD, Miki K, Tamura Y, Nguyen TB, Watanabe S, Hoshiyama M (2003) Sensory perception during sleep in humans: a magnetoencephalograhic study. Sleep Med 4:493-507.

Kealy J, Commins S (2011) The rat perirhinal cortex: a review of anatomy, physiology, plasticity, and function. Prog Neurobiol 93:522-548.

Krom AJ, Marmelshtein A, Gelbard-Sagiv H, Tankus A, Hayat D, Matot I, Strauss I, Fahoum F, Soehle M, Boström J, Mormann F, Fried I, Nir Y (2018) Anesthesia-induced loss of consciousness disrupts auditory responses beyond primary cortex. Available at https://doi.org/10.1101/ 502385.

Lee CM, Osman AF, Volgushev M, Escabí MA, Read HL (2016) Neural spike-timing patterns vary with sound shape and periodicity in three auditory cortical fields. J Neurophysiol 115:1886-1904.

Livingstone MS, Hubel DH (1981) Effects of sleep and arousal on the processing of visual information in the cat. Nature 291:554-561.

MakovS, Sharon O, Ding N, Ben-Shachar M, Nir Y, Zion Golumbic E (2017) Sleep disrupts high-level speech parsing despite significant basic auditory processing. J Neurosci 37:7772-7781.

Massimini M, Ferrarelli F, Huber R, Esser SK, Singh H, Tononi G (2005) Breakdown of cortical effective connectivity during sleep. Science 309: 2228-2232.

Massimini M, Ferrarelli F, Murphy M, Huber R, Riedner B, Casarotto S, Tononi G (2010) Cortical reactivity and effective connectivity during REM sleep in humans. Cogn Neurosci 1:176-183.

McCormick DA, Bal T (1994) Sensory gating mechanisms of the thalamus. Curr Opin Neurobiol 4:550-556.

Murakami M, Kashiwadani H, Kirino Y, Mori K (2005) State-dependent sensory gating in olfactory cortex. Neuron 46:285-296.

Neckelmann D, Ursin R (1993) Sleep stages and EEG power spectrum in relation to acoustical stimulus arousal threshold in the rat. Sleep 16:467477.

Nir Y, Tononi G (2010) Dreaming and the brain: from phenomenology to neurophysiology. Trends Cogn Sci 14:88-100.

Nir Y, Vyazovskiy VV, Cirelli C, Banks MI, Tononi G (2015) Auditory responses and stimulus-specific adaptation in rat auditory cortex are preserved across NREM and REM sleep. Cereb Cortex 25:1362-1378. 
Oudiette D, Paller KA (2013) Upgrading the sleeping brain with targeted memory reactivation. Trends Cogn Sci 17:142-149.

Pelletier JG, Apergis J, Paré D (2004) Low-probability transmission of neocortical and entorhinal impulses through the perirhinal cortex. J Neurophysiol 91:2079-2089.

Peña JL, Pérez-Perera L, Bouvier M, Velluti RA (1999) Sleep and wakefulness modulation of the neuronal firing in the auditory cortex of the guinea pig. Brain Res 816:463-470.

Pereira A, Ribeiro S, Wiest M, Moore LC, Pantoja J, Lin SC, Nicolelis MA (2007) Processing of tactile information by the hippocampus. Proc Natl Acad Sci U S A 104:18286-18291.

Portas CM, Krakow K, Allen P, Josephs O, Armony JL, Frith CD (2000) Auditory processing across the sleep-wake cycle: simultaneous EEG and fMRI monitoring in humans. Neuron 28:991-999.

Profant O, Burianová J, Syka J (2013) The response properties of neurons in different fields of the auditory cortex in the rat. Hear Res 296:51-59.

Quiroga RQ, Nadasdy Z, Ben-Shaul Y (2004) Unsupervised spike detection and sorting with wavelets and superparamagnetic clustering. Neural Comput 16:1661-1687.

Rasch B, Büchel C, Gais S, Born J (2007) Odor cues during slow-wave sleep prompt declarative memory consolidation. Science 315:1426-1429.
Rothschild G, Eban E, Frank LM (2017) A cortical-hippocampal-cortical loop of information processing during memory consolidation. Nat Neurosci 20:251-259.

Sela Y, Vyazovskiy VV, Cirelli C, Tononi G, Nir Y (2016) Responses in rat core auditory cortex are preserved during sleep spindle oscillations. Sleep 39:1069-1082.

Steriade M (2003) Neuronal substrate of sleep and epilepsy. Cambridge, UK: Cambridge UP.

Steriade M, McCarley RW (1990) Brainstem control of wakefulness and sleep. Boston: Springer.

Strauss M, Sitt JD, King JR, Elbaz M, Azizi L, Buiatti M, Naccache L, van Wassenhove V, Dehaene S (2015) Disruption of hierarchical predictive coding during sleep. Proc Natl Acad Sci U S A 112:E1353-E1362.

Vinnik E, Antopolskiy S, Itskov PM, Diamond ME (2012) Auditory stimuli elicit hippocampal neuronal responses during sleep. Front Syst Neurosci 6:49.

Zerbi V, Floriou-Servou A, Markicevic M, Vermeiren Y, Sturman O, Privitera M, von Ziegler L, Ferrari KD, Weber B, De Deyn PP, Wenderoth N, Bohacek J (2019) Rapid reconfiguration of the functional connectome after chemogenetic locus coeruleus activation. Neuron 103:702718.e5. 